\title{
Apelin receptor upregulation in spontaneously hypertensive rat contributes to the enhanced vascular smooth muscle cell proliferation by activating autophagy
}

\author{
Tao Xu ${ }^{1 \#}$, Jian Jia ${ }^{2 \#}, \mathrm{Na} \mathrm{Xu}^{1}$, Chao $\mathrm{Ye}^{3}$, Fen Zheng ${ }^{3}$, Yan Yuan ${ }^{3}$, Guo-Qing Zhu ${ }^{3} \wedge$, Yi-Yang Zhan ${ }^{1}$ \\ ${ }^{1}$ Department of Geriatric Medicine, The First Affiliated Hospital, Nanjing Medical University, Nanjing, China; ${ }^{2}$ Department of General Practice, \\ The First Affiliated Hospital, Nanjing Medical University, Nanjing, China; ${ }^{3}$ Key Laboratory of Targeted Intervention of Cardiovascular Disease, \\ Collaborative Innovation Center of Translational Medicine for Cardiovascular Disease, and Department of Physiology, Nanjing Medical University, \\ Nanjing, China \\ Contributions: (I) Conception and design: T Xu, J Jia, GQ Zhu, YY Zhan; (I) Administrative support: YY Zhan, GQ Zhu; (I) Provision of study \\ materials: T Xu, J Jia, N Xu, C Ye, F Zheng, Y Yuan; (I) Collection and assembly of data: T Xu, J Jia, N Xu, C Ye, F Zheng, GQ Zhu; (I) Data \\ analysis and interpretation: T Xu, J Jia, N Xu, C Ye, F Zheng, GQ Zhu; (I) Manuscript writing: All authors; (I) Final approval of manuscript: All \\ authors. \\ "These authors equally contributed to this work. \\ Correspondence to: Yi-Yang Zhan, PhD. Professor, Department of Geriatric Medicine, The First Affiliated Hospital, Nanjing Medical University, \\ Nanjing 210029, China. Email: yiyangzhan@sina.com; Guo-Qing Zhu, MD, PhD. Professor, Department of Physiology, Nanjing Medical \\ University, 101 Longmian Avenue, Nanjing 211166, China. Email: gqzhucn@njmu.edu.cn.
}

Background: Proliferation of vascular smooth muscle cells (VSMCs) plays a vital role in the progression of vascular remodeling and hypertension. Apelin-13 promotes VSMC proliferation of normal rats. This study was designed to investigate the roles of apelin receptor (APJ) and apelin-13 in VSMC proliferation of hypertension rats and underlying mechanisms.

Methods: Primary VSMCs were obtained from aorta of Wistar-Kyoto rat (WKY) and spontaneously hypertensive rat (SHR). The expressions of apelin and APJ were detected by Western bolt and PCR, as well as immunohistochemistry. VSMC proliferation was evaluated with CCK-8 kit, PCNA protein expression and percentage of EdU-positive cells. Autophagy was determined by the ratio of LC3BII to LC3BI, ATG5 and p62 protein expressions, as well as LC3B immunofluorescence.

Results: APJ expression was increased while apelin expression was reduced in aorta and VSMCs of SHR compared with those of WKY. Exogenous apelin-13 promoted VSMC proliferation and autophagy of both WKY and SHR, which were prevented by APJ antagonist F13A. Blockade of APJ had no significant effects on VSMC proliferation and autophagy of WKY, but attenuated VSMC proliferation and autophagy of SHR. Administration of autophagy inhibitor 3-methyladenine (3-MA) not only attenuated VSMC proliferation of SHR, but prevented apelin-13-induced VSMC proliferation of both WKY and SHR.

Conclusions: Apelin-13 stimulates VSMC proliferation via APJ-mediated enhancement in autophagy. APJ upregulation in SHR contributes to the enhanced VSMC proliferation.

Keywords: Hypertension; apelin receptor (APJ); vascular smooth muscle cells (VSMCs); proliferation; autophagy

Submitted Oct 13, 2020. Accepted for publication Feb 10, 2021.

doi: 10.21037/atm-20-6891

View this article at: http://dx.doi.org/10.21037/atm-20-6891

\footnotetext{
$\wedge$ ORCID: 0000-0002-3132-9592.
} 


\section{Introduction}

Vascular remodeling greatly contributes to the pathogenesis of hypertension, atherosclerosis, diabetic angiopathy, and vascular injury (1-3). Vascular smooth muscle cells (VSMCs) are the primary cell components in the media of arteries. VSMC proliferation plays a vital role in vascular remodeling in hypertension and atherosclerosis (4-6). Intervention of excessive VSMC proliferation serves as a therapeutic target in vascular remodeling and hypertension $(6,7)$.

Apelin receptor (APJ) is a member of the G proteincoupled receptor gene family (8). Although APJ is similar to angiotensin II type 1 receptor $\left(\mathrm{AT}_{1} \mathrm{R}\right)$ in structure, but it displays no affinity for angiotensin II (9). Apelin, a peptide with 77 amino, is identified as an endogenous ligand for the APJ (8). Apelin is processed to produce bioactive peptides consisting of 12, 13, 17 and 36 amino acids, which is called apelin-12, apelin-13, apelin-17 and apelin-36, respectively $(10,11)$. Apelin-13 shows the highest biological activity among those forms (12). Apelin/APJ system plays important roles in the cardiovascular system under both normal and pathophysiological conditions (13-15). It is known that apelin promotes VSMC proliferation in normal or atherosclerotic animals (15-18). Apelin-13 and APJ in the paraventricular nucleus are involved in vasopressin release and sympathetic activation in spontaneously hypertensive rat (SHR) (19). However, the roles of peripheral vascular apelin/APJ system in VSMC proliferation in hypertension remain unknown.

Autophagy is a dynamic process that participates in cellular homeostasis by regulating cell survival and death (20). Intracellular organelles or proteins are delivered to lysosomes, which regulate cell survival through lysosomemediated degradation (21). Hypertension and several vascular diseases are closely linked to autophagy dysfunction $(22,23)$. Autophagy at a low level maintains homeostasis and provides energy and nutrients for cells through degradation of damaged organelles, while excessive autophagy damages cells and even causes cell death (24). Vascular autophagy is involved in physiological processes and some diseases relate to vascular structure and function (25). It has been found that AMPK activation prevents pulmonary arterial hypertension induced by monocrotaline via inhibiting NF$\kappa \mathrm{B}$-mediated autophagy (26). TP53-induced glycolysis and apoptosis regulator attenuates VSMC autophagy of pulmonary artery to prevent pulmonary hypertension (27). However, the role of autophagy in VSMC proliferation and its relation to apelin/APJ system in hypertension are still not elucidated. The present study aims at determining the roles of apelin/APJ in autophagy and proliferation of VSMCs in Wistar-Kyoto rat (WKY) and SHR.

We present the following article in accordance with the ARRIVE reporting checklist (available at http://dx.doi. org/10.21037/atm-20-6891).

\section{Methods}

\section{Experimental animals}

Experiments were designed according to the standards of the Guide for the Care and Use of Laboratory Animals published by the US National Institutes of Health (NIH, 8th edition, 2011). The surgical procedures and experimental protocol were approved by the Experimental Animal Care and Use Committee of Nanjing Medical University (No.: IACUC-1811017). SHR is widely used as a model of hypertension, and WKY, derived from the same ancestral Wistar rat as the SHR, is normotensive and used as the closest genetic control for the SHR. Male WKY $(n=12)$ and SHR $(n=12)$ aged at 9 weeks weighing 200-250 g were purchased from Vital River Laboratory Animal Technology Co., Ltd. (Beijing, China). Systolic blood pressure (SBP) for SHR was higher than $150 \mathrm{~mm} \mathrm{Hg}$ in the present study. Rats were exposed to a temperaturecontrolled room for a $12 \mathrm{~h}$ light/12 h dark cycle. The rats were randomly fed with a standard chow and tap water. Rat was euthanized at the end of experiments with intravenous injection of pentobarbital sodium $(200 \mathrm{mg} / \mathrm{kg})$.

\section{Cell culture}

VSMCs were isolated from thoracic aorta of WKY and SHR and cultured as we reported previously (5). Simply, the cells were cultured in DMEM containing $10 \%$ fetal bovine serum (FBS), $100 \mathrm{IU} \cdot \mathrm{mL}^{-1}$ penicillin and $100 \mathrm{mg} \cdot \mathrm{mL}^{-1}$ streptomycin in humidified containing $5 \% \mathrm{CO}_{2}$ at $37{ }^{\circ} \mathrm{C}$. VSMCs were identified with negative PECAM-1 (marker of endothelial cells) and vimentin (marker of fibroblasts), and positive $\alpha$-SMA (marker of VSMCs). VSMCs from the $3^{\text {rd }}$ to the $5^{\text {th }}$ passages were used in the present study. The VSMCs were incubated $24 \mathrm{~h}$ after PBS or apelin-13 (1 $\mu \mathrm{M}$, MedChem Express, Monmouth Junction, NJ, USA) treatments.

\section{Evaluation of cell proliferation}

Cell proliferation was evaluated with cell counting kit-8 
(CCK-8) kit, expression of proliferating cell nuclear antigen (PCNA) protein and percentage of 5-ethynyl2 '-deoxyuridine (EdU)-positive cells as we previously reported (5). Absorbance was measured at $450 \mathrm{~nm}$ with a microplate reader (ELX800, BioTek, Vermont, USA). PCNA protein expression was determined with Western blotting. DNA synthesis was examined with EdU incorporation assay to evaluate the VSMC proliferation with a Cell-Light ${ }^{\mathrm{TM}}$ EdU Apollo ${ }^{\circledR} 567$ In Vitro Imaging Kit (RiboBio, Guangzhou, China). Images were taken with a fluorescence microscopy (DP70, Olympus Optical, Tokyo, Japan). The number of EdU-positive cells was normalized by total number of cells.

\section{Immunobistochemistry}

Immunohistochemistry was used to detect APJ and apelin expressions in aorta of WKY and SHR. The aorta was fixed in $4 \%$ paraformaldehyde, embedded in paraffin, and then cut into $10 \mu \mathrm{m}$ sections. The deparaffinized and rehydrated sections were incubated with primary anti-APJ antibody (1:100) or anti-apelin antibody $(1: 100)$ overnight at $4{ }^{\circ} \mathrm{C}$, and then incubated with horseradish peroxidase-conjugated goat anti-rabbit antibody.

\section{Immunofluorescent analysis for $L C 3 B$}

VSMCs were grown on glass coverslips in 6-well plates and fixed with $4 \%$ paraformaldehyde for $20 \mathrm{~min}$. The cells were rinsed with $1 \times \mathrm{PBS}$ for three times and permeabilized with $0.1 \%$ Triton $\mathrm{X}-100$ for $5 \mathrm{~min}$. After blocking in $1 \%$ BSA at room temperature for $1 \mathrm{~h}$, cells were incubated with microtube-associated protein-1 light chain-3B (LC3B) antibody (1:100) and $\alpha$-SMA antibody $(1: 100)$ overnight. The cells were rinsed with $1 \times \mathrm{PBS}$ and incubated with the secondary fluorescein anti-rabbit $\operatorname{IgG}(1: 1,000)$ and antimouse $\operatorname{IgG}(1: 1,000)$ for $1 \mathrm{~h}$. The nuclei were stained with DAPI (Southern Biotech, Birmingham, AL, USA), a bluefluorescent DNA stain.

\section{$R N A$ extraction and $q R T-P C R$}

Total RNA was exacted from thoracic aorta and VSMCs with a Trizol reagent (Life Technologies, Gaithersburg, MD, USA). Then, $1 \mu \mathrm{g}$ total RNA was reversed to cDNA using the PrimeScript ${ }^{\circledR}$ RT reagent Kits (Takara, Otsu, Shiga, Japan) and StepOnePlus ${ }^{\mathrm{TM}}$ Real-Time PCR System (Applied Biosystems, Foster City, CA, USA). The quantitative reactions were made with SYBR Green (Takara Biotechnology Co., Ltd., Tokyo, Japan) by StepOnePlus ${ }^{\mathrm{TM}}$ system. GAPDH was used as the internal control. All primers used for qRT-PCR are listed in a table (Table S1).

\section{Western blot analysis}

Total protein was extracted from VSMCs and thoracic aorta respectively. Then, $30 \mu \mathrm{g}$ total proteins were separated with SDS-PAGE and transferred to PVDF membrane. The membranes were blocked with $5 \%$ defatted milk in TBST, and then incubated with primary antibody overnight at $4{ }^{\circ} \mathrm{C}$. The Enhanced Chemiluminescence Detection Kit (Thermo Fisher Scientific, USA) was used to visualize protein bands. All the antibodies used for immunofluorescent and Western blot analysis are listed in a table (Table S2).

\section{Statistical analysis}

Data are expressed as mean \pm SE. Student's unpaired $t$-test was used to compare the difference between two groups. One-way or two-way ANOVA followed by post hoc Bonferroni test was employed for multiple comparisons. Statistical significance was considered as $\mathrm{P}<0.05$.

\section{Results}

\section{APf and apelin expressions in the aorta and VSMCs}

APJ mRNA level was increased but the apelin mRNA level was not significantly altered in aorta of SHR compared with those of WKY (Figure 1A). APJ protein was upregulated $(+73.8 \%)$ while apelin was downregulated $(-28.1 \%)$ in the aorta of SHR compared with those of WKY (Figure 1B). Immunohistochemistry analysis further confirmed that the APJ expression was increased (Figure 1C) while apelin expression was reduced in the aorta of SHR (Figure 1D). Consistently, APJ protein expression was increased $(+63.7 \%)$, but apelin protein expression was reduced $(-23.0 \%)$ in VSMCs of SHR compared with those of WKY (Figure 2A). These findings suggest that apelin/APJ may play an important role in regulating VSMCs.

\section{Effects of apelin-13 and F13A on VSMC proliferation}

VSMC proliferation was evaluated with CCK-8 kit, PCNA protein expression and percentage of EdU-positive cells. Apelin-13 promoted VSMC proliferation of both WKY 


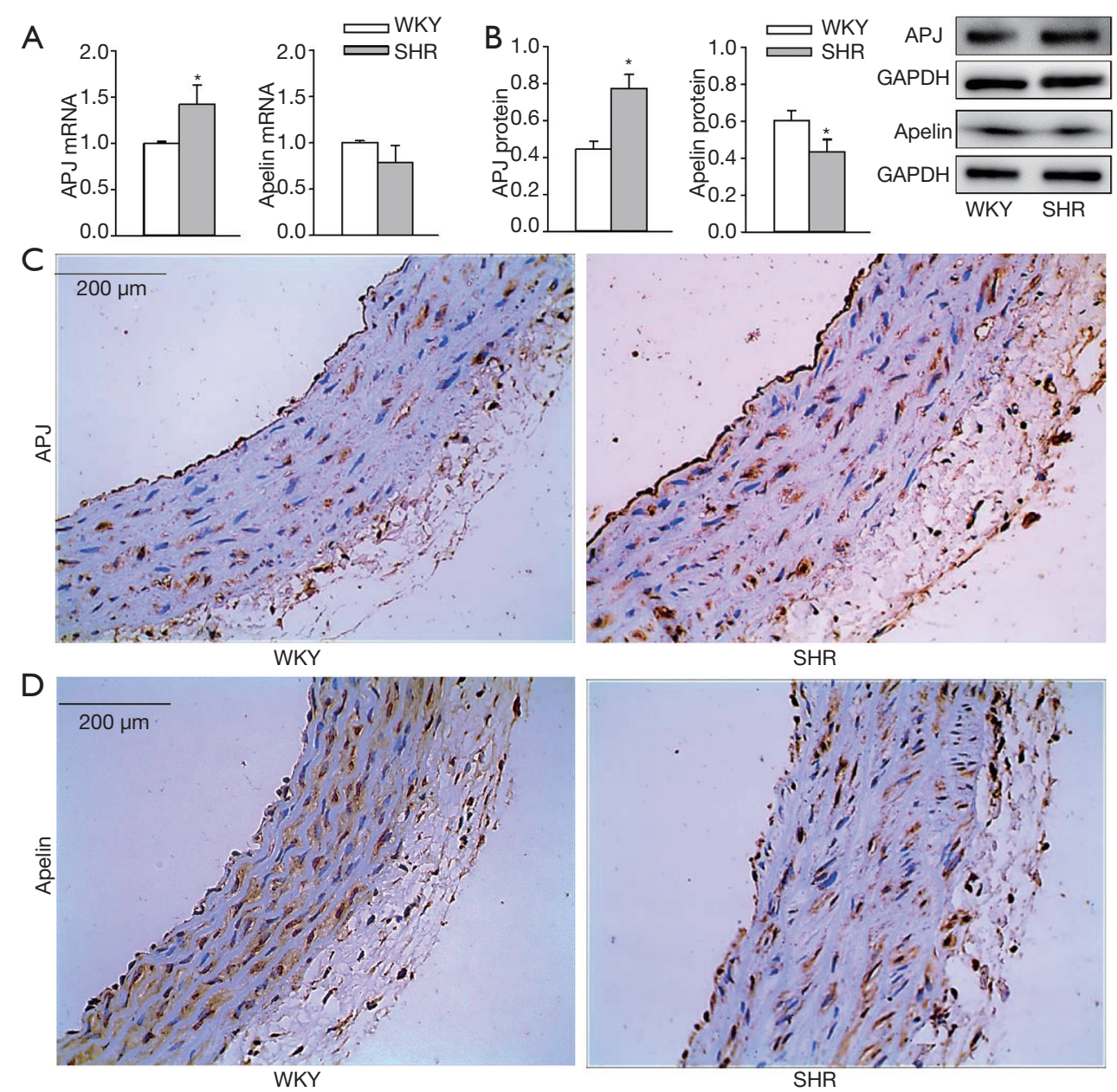

Figure 1 APJ and apelin expressions in aorta of WKY and SHR. (A) APJ and apelin mRNA expressions. (B) APJ and apelin protein expressions. (C) Immunohistochemistry of aorta sections for APJ (brown color). (D) Immunohistochemistry of aorta sections for apelin (brown color). Values are mean \pm SE. *, $\mathrm{P}<0.05$ vs. WKY. $\mathrm{n}=6$. WKY, Wistar-Kyoto rat; SHR, spontaneously hypertensive rat; APJ, apelin receptor.

and SHR. APJ antagonist F13A had no significant effects on VSMC proliferation of WKY, but inhibited VSMC proliferation of SHR. Furthermore, F13A prevented apelin13-induced VSMC proliferation in VSMCs of both WKY and SHR (Figure 2B,C,D,E). These results indicated that apelin-13 stimulates VSMC proliferation via APJ in both WKY and SHR. The upregulation of APJ in VSMCs of SHR contributes to the enhanced VSMC proliferation in SHR.

\section{Effects of apelin-13 and F13A on autophagy of VSMCs}

Increased LC3B II production from LC3BI and ATG5 protein expression as well as reduced $\mathrm{p} 62$ protein expression are markers of autophagy (28-30). Apelin-13 promoted autophagy in VSMCs of both WKY and SHR. APJ antagonist F13A had no significant effects on autophagy in VSMCs of WKY, but attenuated autophagy in VSMCs of SHR. Furthermore, F13A prevented apelin-13induced autophagy in VSMCs of both WKY and SHR (Figure $3 A, B, C$ ). The findings were further supported by the triple immunofluorescence staining for LC3B (Figure $4 A, B$ ). These results indicated that apelin-13 promotes autophagy via APJ in VSMCs of WKY and SHR. The increased APJ expression in VSMCs of SHR is involved in the enhanced autophagy in VSMCs of SHR. 

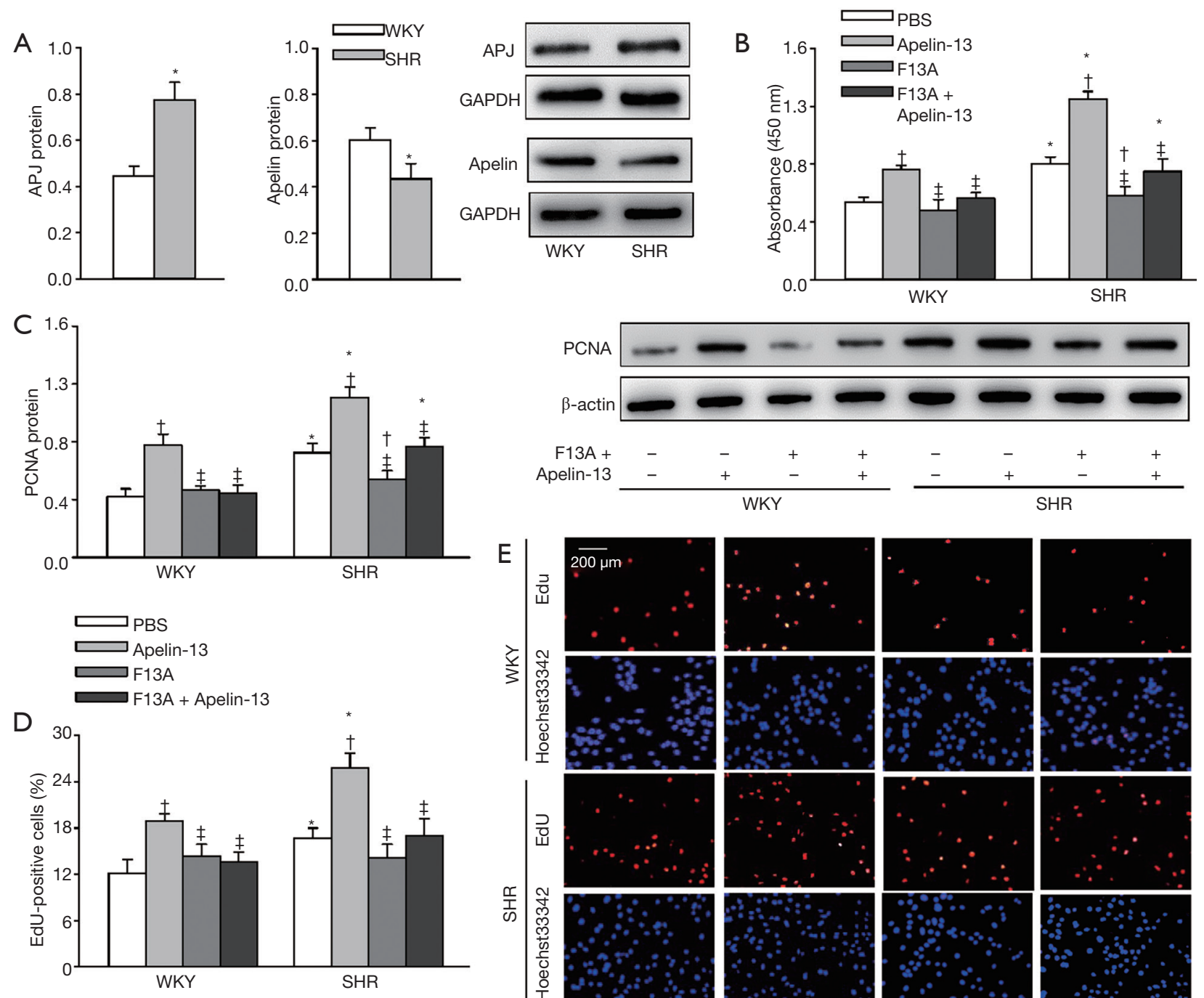

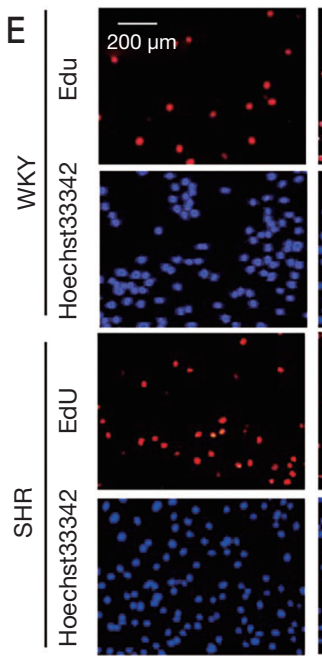

PBS

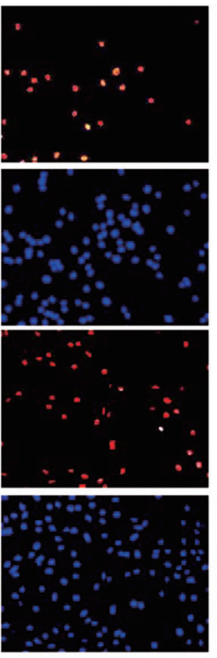

Apelin-13

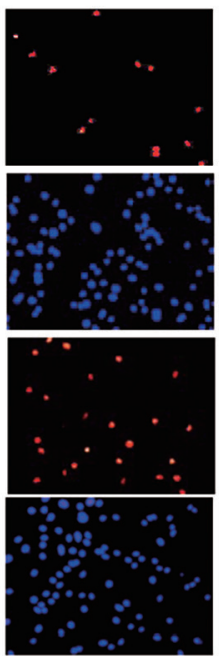

F13A

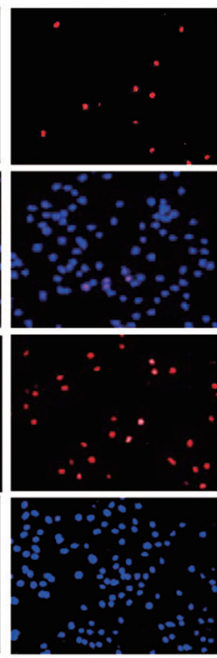

$\mathrm{F} 13 \mathrm{~A}+$ Apelin-13

Figure 2 APJ and apelin protein expressions in VSMCs and the effects of apelin-13 and APJ receptor antagonist F13A on VSMC proliferation of WKY and SHR. (A) APJ and apelin protein expressions in VSMCs of WKY and SHR. (B) VSMC proliferation evaluated with CCK-8 kit. (C) VSMC proliferation evaluated with PCNA protein expression. (D) VSMC proliferation evaluated with the percentage of EdU-positive cells. (E) Representative images of EdU staining. Red, EdU-positive cells. Blue, Hoechst 33342 was used to label doublestranded DNA and thus to visualize nuclei. The measurements were carried out $24 \mathrm{~h}$ after PBS, apelin-13 $(1 \mu \mathrm{M})$, or F13A $(1 \mu \mathrm{M})$ treatment. Values are mean $\pm \mathrm{SE}$. *, $\mathrm{P}<0.05$ vs. WKY; $\dagger, \mathrm{P}<0.05$ vs. $\mathrm{PBS} ; \ddagger, \mathrm{P}<0.05$ vs. Apelin-13. $\mathrm{n}=6$. VSMC, vascular smooth muscle cell; F13A, APJ antagonist; CCK-8, cell counting kit-8; PCNA, proliferating cell nuclear antigen; EdU, 5-ethynyl-2'-deoxyuridine.

\section{Time-effects of apelin-13 on VSMC autophagy and proliferation}

Apelin-13 time-relatedly promoted VSMC autophagy and proliferation in WKY and SHR. The autophagy was significantly enhanced at the 8 th $\mathrm{h}$ after application of apelin-13 (Figure $5 A, B$ ), while the proliferation was significantly enhanced at the 16 th $\mathrm{h}$ after application of apelin-13 (Figure 5C). The results indicate that the effects of apelin-13 on autophagy occur earlier than those on proliferation. 

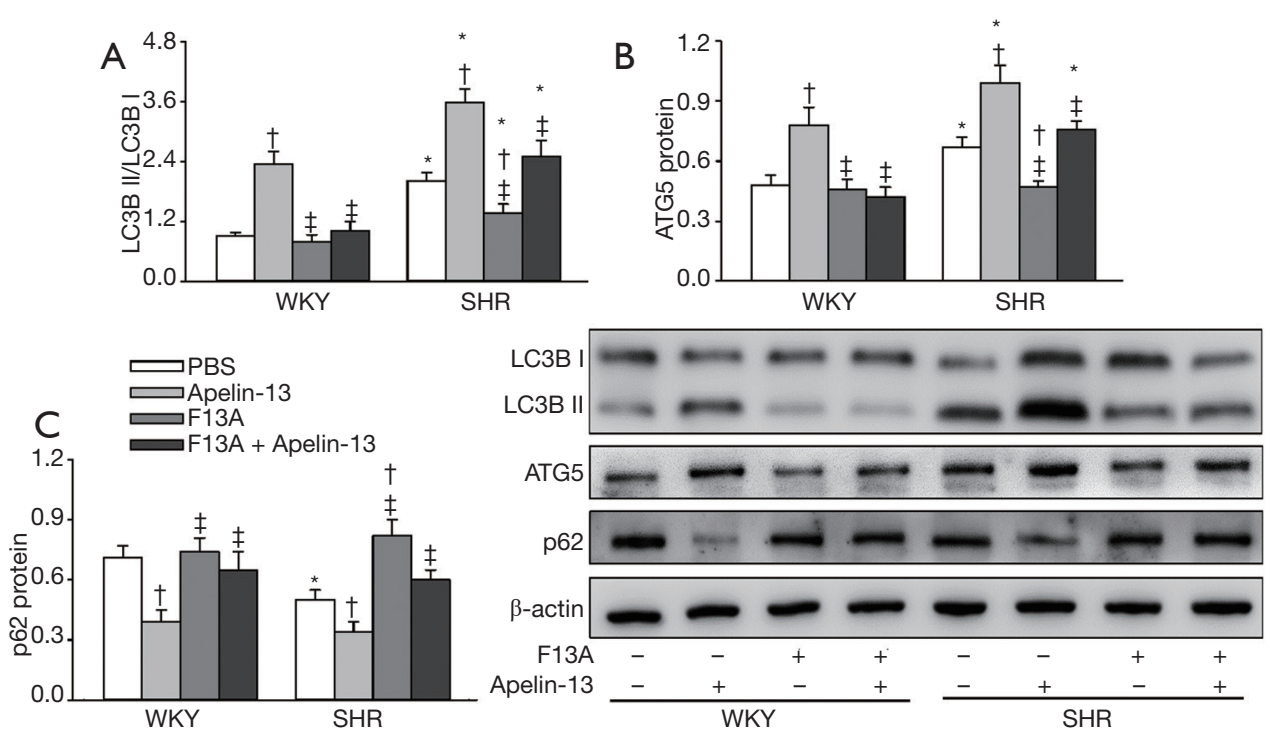

Figure 3 Effects of apelin-13 and apelin receptor antagonist F13A on VSMC autophagy of WKY and SHR. Autophagy determined by LC3B, ATG5 and p62 protein expressions. (A) Ratio of LC3B II to LC3B I. (B) ATG5 protein expression in VSMCs. (C) p62 protein expression in VSMCs. The measurements were carried out $24 \mathrm{~h}$ after PBS, apelin-13 $(1 \mu \mathrm{M})$ or F13A $(1 \mu \mathrm{M})$ treatment. Values are mean $\pm \mathrm{SE} .{ }^{*}, \mathrm{P}<0.05$ vs. WKY; †, $\mathrm{P}<0.05$ vs. $\mathrm{PBS} ;$ ‡, $\mathrm{P}<0.05$ vs. Apelin-13. $\mathrm{n}=6$. LC3B, microtube-associated protein-1 light chain-3B; ATG5, autophagy related protein 5 .

\section{Effects of 3-MA on apelin-induced VSMC proliferation}

3-Methyladenine (3-MA) is a widely used autophagy inhibitor (31-33). 3-MA had no significant effects on VSMC proliferation of WKY, but inhibits the enhanced VSMC proliferation of SHR. More importantly, apelin-induced VSMC proliferation was prevented by 3-MA treatment (Figure $6 A, B, C, D)$. These data indicated that apelin promotes VSMC proliferation at least partially mediated by enhancing autophagy in VSMCs.

\section{Discussion}

VSMC proliferation is important for vascular remodeling in hypertension (34-36). Vascular remodeling may initially be adaptive in hypertension, but eventually becomes maladaptive and contributes to the development of hypertension and occurrence of its complications (37-39). The primary novel findings are that APJ upregulation in SHR greatly contributes to the VSMC proliferation, and apelin-13 promotes VSMC proliferation by APJ-mediated activation of autophagy in both WKY and SHR. Blockade of APJ attenuates VSMC autophagy and proliferation, and inhibition of autophagy suppresses VSMC proliferation in SHR and prevents apelin-13-induced VSMC proliferation in both WKY and SHR. These findings suggest that APJ in VSMCs may be a therapeutic target for inhibiting VSMC autophagy and proliferation in hypertension.

APJ is an orphan G protein-coupled receptor and apelin is the endogenous ligand of APJ. Apelin/APJ system is widely distributed in many tissues and cells including VSMCs (15). It is known that apelin-13 promotes VSMC proliferation in VSMCs of normal animals (40). However, the roles of apelin/APJ in VSMC proliferation of hypertension remain unknown. We found that APJ expression was increased in VSMCs of SHR as well as in the aorta of SHR. APJ antagonist inhibited VSMC proliferation of SHR and prevented apelin-13-induced VSMC proliferation of both WKY and SHR. The findings indicate that the upregulation of APJ plays a crucial role in the enhanced VSMC proliferation in SHR. It is noted that apelin expression was slightly reduced in VSMCs of SHR as well as in the aorta of SHR. The downregulation of apelin in the SHR might be a compensatory protective mechanism to avoid the roles of excessive APJ activation in VSMC proliferation.

Autophagy is crucial for lysosomal degradation and recycling process in cells, which is responsible for maintaining the homeostasis of cell survival and cell death 


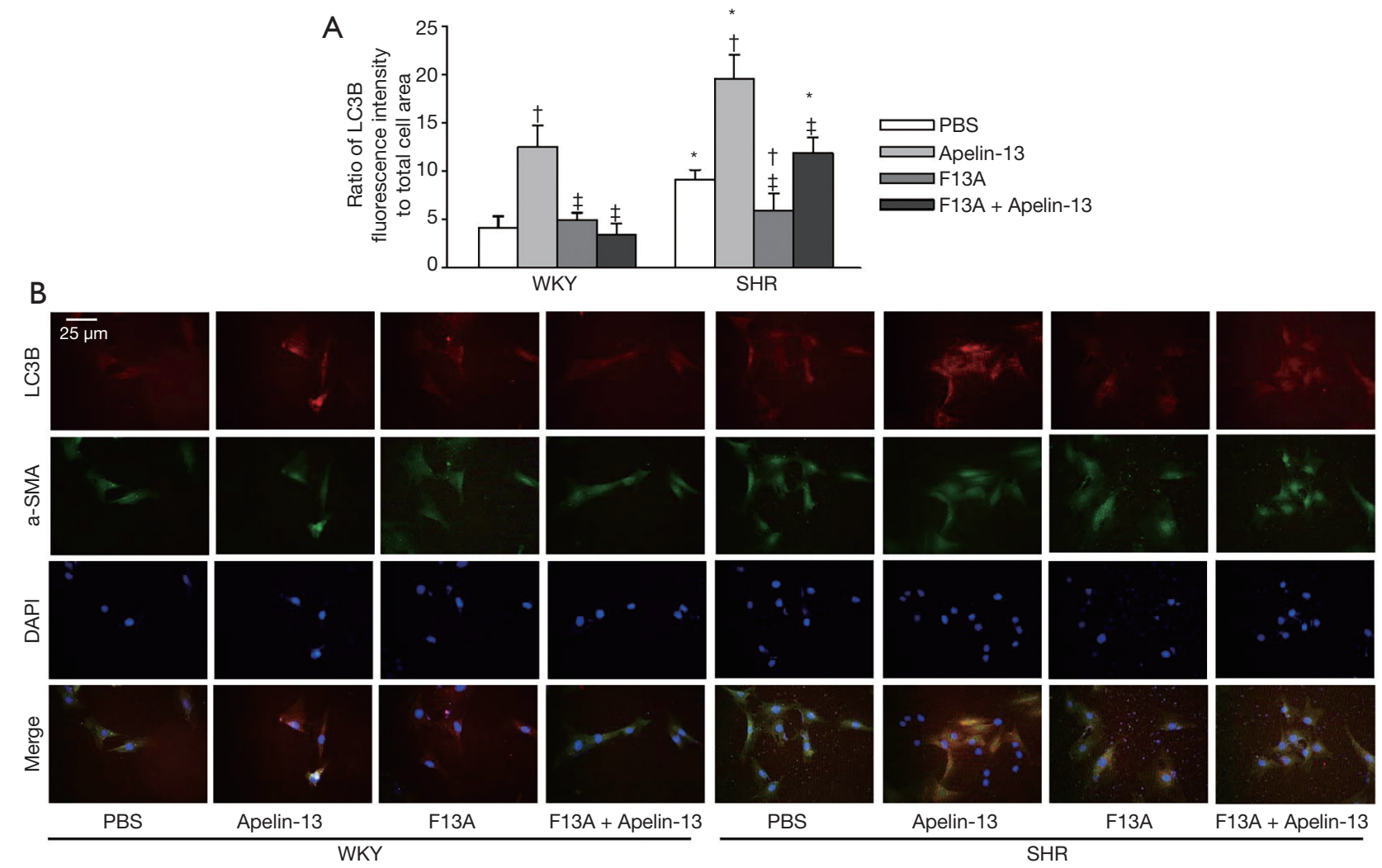

Figure 4 Effects of apelin-13 on LC3B expressions in VSMCs of WKY and SHR. LC3B expression was determined with immunofluorescence staining. The measurements were carried out $24 \mathrm{~h}$ after PBS, apelin-13 ( $1 \mu \mathrm{M})$ or F13A (1 $\mu \mathrm{M})$ treatment. (A) Relative fluorescence intensity of LC3B normalized with cell area. (B) Representative images of triple immunofluorescence staining for LC3B (red), $\alpha$-SMA (green) and DAPI (blue). The $\alpha$-SMA and DAPI staining were respectively used to show VSMC cells and nuclei. Values are mean \pm SE. *, $\mathrm{P}<0.05$ vs. WKY; †, $\mathrm{P}<0.05$ vs. PBS; $\neq, \mathrm{P}<0.05$ vs. Apelin-13. $\mathrm{n}=5$. $\alpha$-SMA, $\alpha$-smooth muscle actin; DAPI, 4',6-diamidino-2phenylindole.

as well as the cellular function. It is very important for waste removal and structural reconstruction in cells and is involved in the pathogenesis of hypertension (22). Generally, basal or mild autophagy level is beneficial for cellular homeostasis and cell survival, while moderate autophagy promotes cellular phenotype change, proliferation and migration, and excessive autophagy causes severe self-digestion and even cell death. Therefore, moderate or excessive autophagy is involved in vascular diseases including atherosclerosis and restenosis (41). We found that autophagy was enhanced in VSMCs of SHR, which was attenuated by blockade of APJ. The roles of apelin-13 in promoting VSMC autophagy of WKY and SHR were prevented by the blockade of APJ. More importantly, autophagy inhibitor attenuated VSMC proliferation of SHR, and prevents apelin-13-induced VSMC proliferation of both WKY and SHR. These findings provide solid evidences that apelin/APJ activation promotes VSMC proliferation by activating VSMC autophagy in SHR, which is supported by the fact that TP53-induced glycolysis and apoptosis regulator inhibits autophagy of pulmonary artery smooth muscle cells to prevent pulmonary arterial hypertension (27).

Recent studies have shown the complicated signal pathways of apelin/APJ in VSMC proliferation of normal animals. Apelin in VSMCs activates PI3K/Akt, Egr-1, 14-33/Raf-1, and NOX4/ROS, which accelerates the conversion from $\mathrm{G} 1$ phase to $\mathrm{S}$ phase during mitosis, and transmits the signal from ERK to cyclin D1, and then enhances proliferation of VSMCs (15). Furthermore, PINK1/Parkinmediated mitophagy stimulates apelin-13-induced VSMC proliferation via activation of AMPK $\alpha$, and exacerbates atherosclerotic lesion (42), suggesting that the roles of 
A

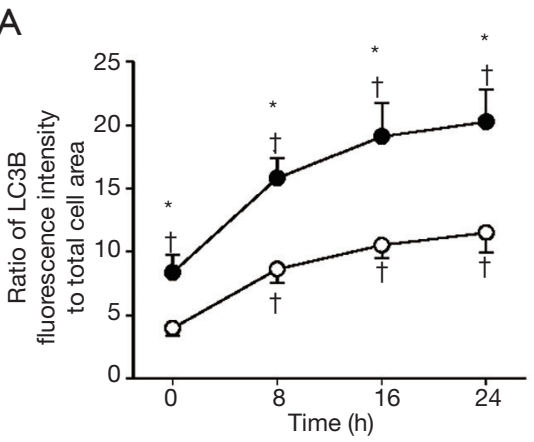

-WHKY
$\longrightarrow$ SHR

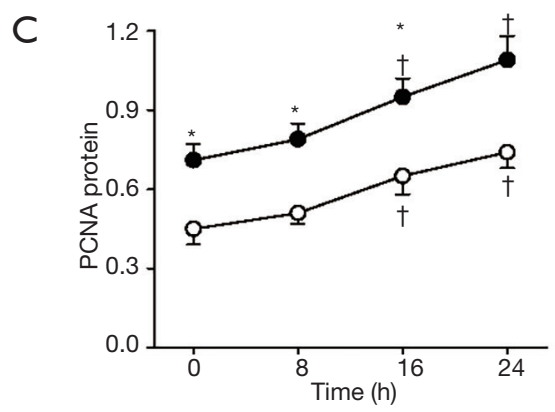

B
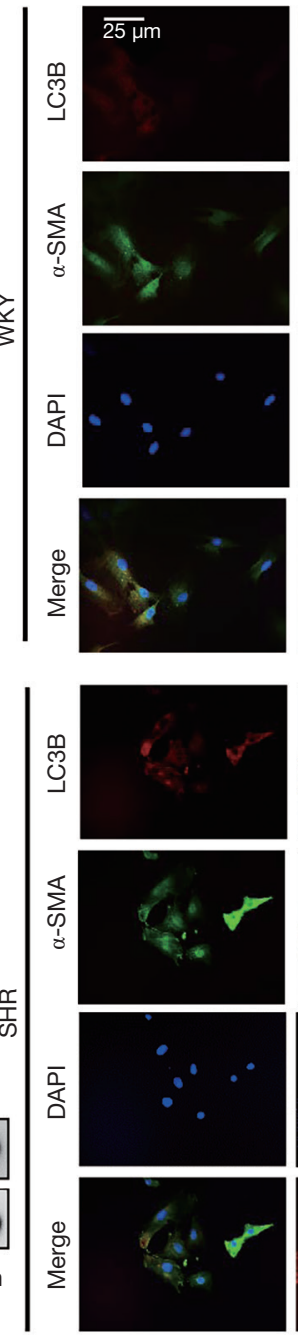

$\mathrm{Oh}$
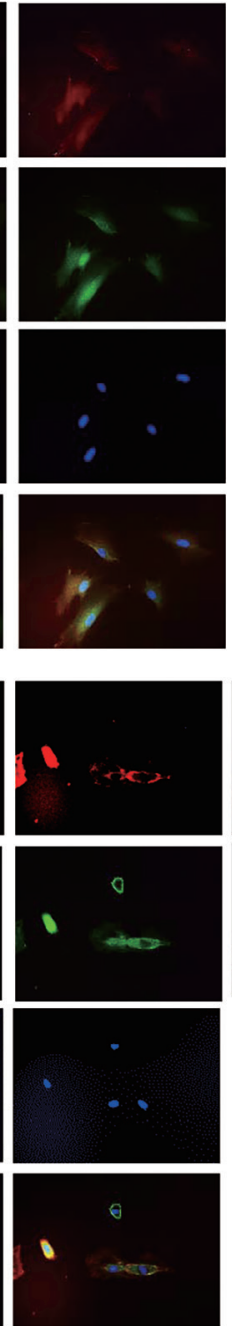

$8 \mathrm{~h}$
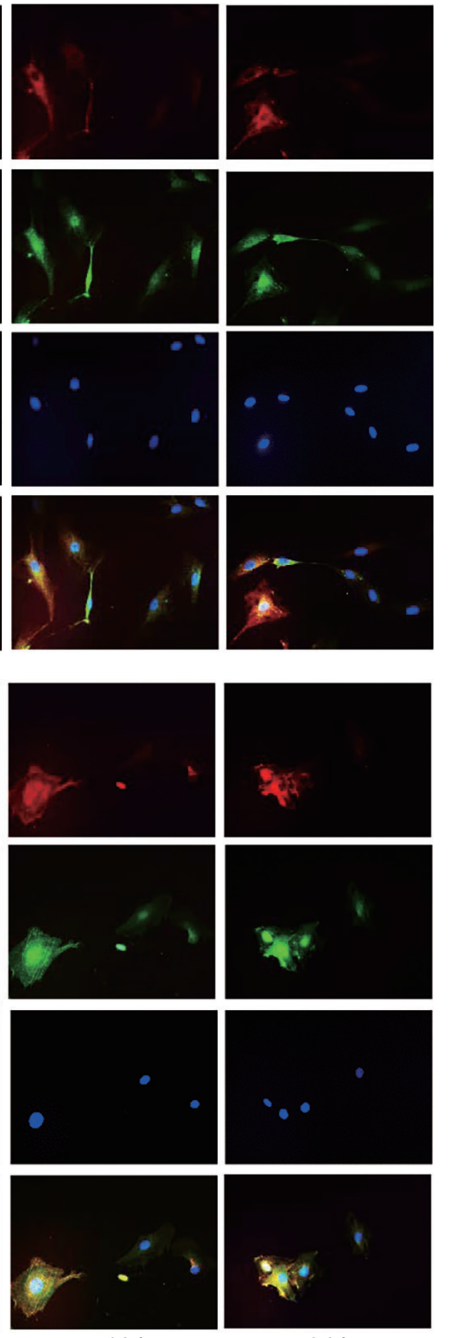

$16 \mathrm{~h}$

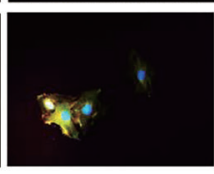

$24 \mathrm{~h}$

Figure 5 Time-effects of apelin-13 on LC3B and PCNA expressions in VSMCs of WKY and SHR. LC3B expression was determined with immunofluorescence staining. PCNA expression was examined with Western Blot. The measurements were respectively examined $0,8,16$ and $24 \mathrm{~h}$ after apelin-13 (1 $\mathrm{MM})$ treatment. (A) Relative fluorescence intensity of LC3B normalized with cell area. (B) Representative images of triple immunofluorescence staining for LC3B (red), $\alpha$-SMA (green) and DAPI (blue). The $\alpha$-SMA and DAPI staining were respectively used to show VSMC cells and nuclei. (C) VSMC proliferation evaluated with PCNA protein expression. Values are mean $\pm \mathrm{SE}$. * $\mathrm{P}<0.05$ vs. WKY; †, $\mathrm{P}<0.05$ vs. 0 h. $\mathrm{n}=4$.

apelin in promoting VSMC proliferation is mediated by AMPK $\alpha /$ PINK1/parkin-autophagy signal pathway (15). However, the primary signal pathways of apelin/APJ in VSMC autophagy and proliferation in SHR remain further investigation.

\section{Conclusions}

Apelin-13 stimulates VSMC proliferation via APJ- dependent autophagy activation in WKY and SHR. Increased APJ expression contributes to VSMC proliferation in SHR. Blockade of APJ inhibits VSMC autophagy and proliferation in SHR, and inhibition of autophagy attenuates VSMC proliferation in SHR and apelin-13-induced VSMC proliferation of both WKY and SHR. Intervention of APJ in VSMCs might be a strategy for attenuating VSMC proliferation and autophagy in hypertension. 


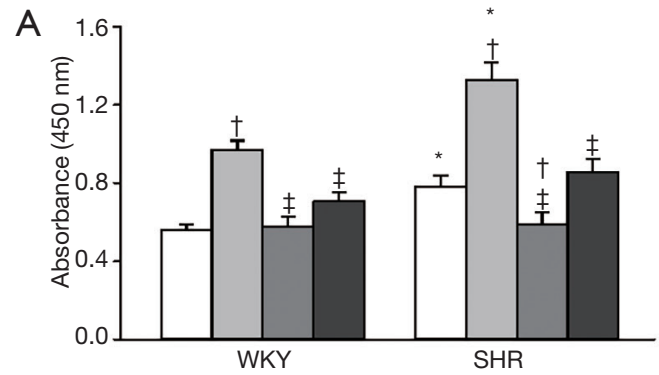

B
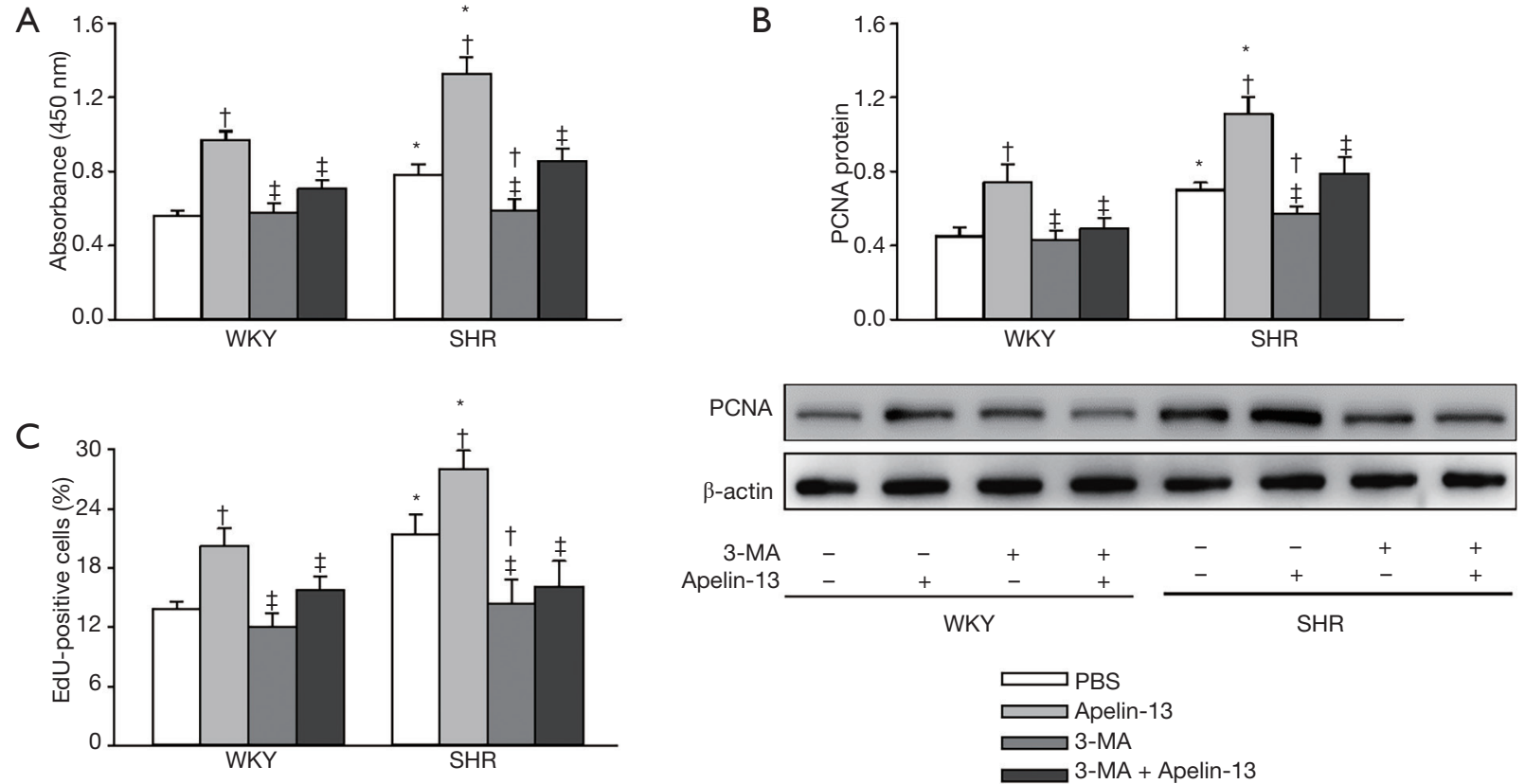

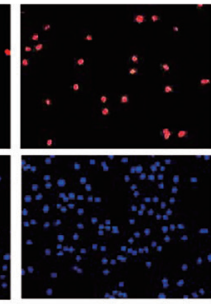

Apelin-13

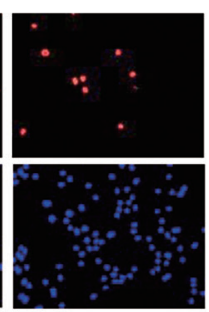

3-MA

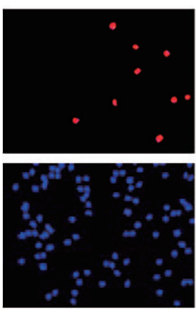

3-MA + Apelin-13

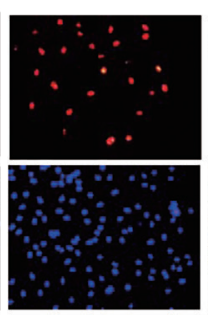

PBS

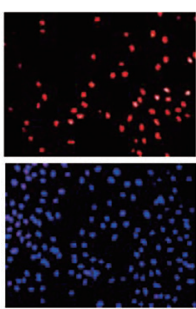

Apelin-13

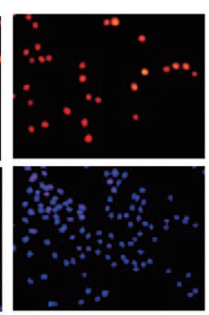

3-MA

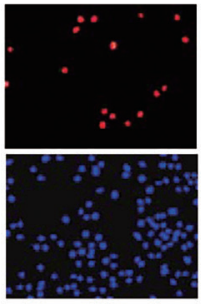

3-MA + Apelin-13

Figure 6 Effects of autophagy inhibitor 3-MA on apelin-13-induced VSMC proliferation of WKY and SHR. (A) VSMC proliferation evaluated with CCK-8 kit. (B) VSMC proliferation evaluated with PCNA protein expression. (C) VSMC proliferation evaluated with the percentage of EdU-positive cells. (D) Representative images of EdU staining. Red, EdU-positive cells. Blue, Hoechst 33342 was used to label double-stranded DNA and thus to visualize nuclei. The measurements were carried out $24 \mathrm{~h}$ after PBS, apelin-13 (1 $\mu \mathrm{M})$, or $3-\mathrm{MA}$ $(5 \mathrm{mM})$ treatment. Values are mean $\pm \mathrm{SE}$. * $\mathrm{P}<0.05$ vs. WKY; $\dagger, \mathrm{P}<0.05$ vs. $\mathrm{PBS} ;$ †, $\mathrm{P}<0.05$ vs. Apelin-13. $\mathrm{n}=6.3-\mathrm{MA}, 3-\mathrm{methyladenine}$.

\section{Acknowledgments}

Funding: This work was supported by Natural Science Foundation of Jiangsu Province of China (BK20191494), Six Talent Peaks Project in Jiangsu Province (WSN024), and National Natural Science Foundation of China (32071106, 31871148).

\section{Footnote}

Reporting Checklist: The authors have completed the ARRIVE reporting checklist. Available at http://dx.doi. org/10.21037/atm-20-6891
Data Sharing Statement: Available at http://dx.doi. org/10.21037/atm-20-6891

Conflicts of Interest: All authors have completed the ICMJE uniform disclosure form (available at http://dx.doi. org/10.21037/atm-20-6891). The authors have no conflicts of interest to declare.

Ethical Statement: The authors are accountable for all aspects of the work in ensuring that questions related to the accuracy or integrity of any part of the work are appropriately investigated and resolved. Experiments were 
performed under a project license (No.: IACUC-1811017) granted by the Experimental Animal Care and Use Committee of Nanjing Medical University, in compliance with the Guide for the Care and Use of Laboratory Animals published by the US National Institutes of Health $(\mathrm{NIH}$, 8th edition, 2011).

Open Access Statement: This is an Open Access article distributed in accordance with the Creative Commons Attribution-NonCommercial-NoDerivs 4.0 International License (CC BY-NC-ND 4.0), which permits the noncommercial replication and distribution of the article with the strict proviso that no changes or edits are made and the original work is properly cited (including links to both the formal publication through the relevant DOI and the license). See: https://creativecommons.org/licenses/by-nc-nd/4.0/.

\section{References}

1. Rizzoni D, Agabiti RE. Small artery remodeling in hypertension and diabetes. Curr Hypertens Rep 2006;8:90-5.

2. Ponticos M, Smith BD. Extracellular matrix synthesis in vascular disease: hypertension, and atherosclerosis. J Biomed Res 2014;28:25-39.

3. Xu J, Shi GP. Vascular wall extracellular matrix proteins and vascular diseases. Biochim Biophys Acta 2014;1842:2106-19.

4. Chen D, Zang YH, Qiu Y, et al. BCL6 attenuates proliferation and oxidative stress of vascular smooth muscle cells in hypertension. Oxid Med Cell Longev 2019;2019:5018410.

5. Ren XS, Tong Y, Qiu Y, et al. MiR155-5p in adventitial fibroblasts-derived extracellular vesicles inhibits vascular smooth muscle cell proliferation via suppressing angiotensin-converting enzyme expression. J Extracell Vesicles 2019;9:1698795.

6. Wang Y, Zhang X, Gao L, et al. Cortistatin exerts antiproliferation and antimigration effects in vascular smooth muscle cells stimulated by Ang II through suppressing ERK1/2, p38 MAPK, JNK and ERK5 signaling pathways. Ann Transl Med 2019;7:561.

7. Yoshizumi M, Kyotani Y, Zhao J, et al. Targeting the mitogen-activated protein kinase-mediated vascular smooth muscle cell remodeling by angiotensin II. Ann Transl Med 2020;8:157.

8. Tatemoto K, Hosoya M, Habata Y, et al. Isolation and characterization of a novel endogenous peptide ligand for the human APJ receptor. Biochem Biophys Res Commun 1998;251:471-6.

9. O'Dowd BF, Heiber M, Chan A, et al. A human gene that shows identity with the gene encoding the angiotensin receptor is located on chromosome 11. Gene 1993;136:355-60.

10. Cano Martínez LJ, Coral Vazquez RM, Mendez JP, et al. Serum concentrations of apelin-17 isoform vary in accordance to blood pressure categories in individuals with obesity class 3. Clin Exp Hypertens 2019;41:168-73.

11. Mesmin C, Dubois M, Becher F, et al. Liquid chromatography/tandem mass spectrometry assay for the absolute quantification of the expected circulating apelin peptides in human plasma. Rapid Commun Mass Spectrom 2010;24:2875-84.

12. Pitkin SL, Maguire JJ, Bonner TI, et al. International Union of Basic and Clinical Pharmacology. LXXIV. Apelin receptor nomenclature, distribution, pharmacology, and function. Pharmacol Rev 2010;62:331-342.

13. Huang Z, He L, Chen Z, et al. Targeting drugs to APJ receptor: From signaling to pathophysiological effects. J Cell Physiol 2018;234:61-74.

14. Zhong JC, Zhang ZZ, Wang W, et al. Targeting the apelin pathway as a novel therapeutic approach for cardiovascular diseases. Biochim Biophys Acta Mol Basis Dis 2017;1863:1942-50.

15. Liu W, Yan J, Pan W, et al. Apelin/Elabela-APJ: a novel therapeutic target in the cardiovascular system. Ann Transl Med 2020;8:243.

16. Li L, Li L, Xie F, et al. Jagged-1/Notch3 signaling transduction pathway is involved in apelin-13-induced vascular smooth muscle cells proliferation. Acta Biochim Biophys Sin (Shanghai) 2013;45:875-81.

17. Liu C, Su T, Li F, et al. PI3K/Akt signaling transduction pathway is involved in rat vascular smooth muscle cell proliferation induced by apelin-13. Acta Biochim Biophys Sin (Shanghai) 2010;42:396-402.

18. Li F, Li L, Qin X, et al. Apelin-induced vascular smooth muscle cell proliferation: the regulation of cyclin D1. Front Biosci 2008;13:3786-92.

19. Zhang F, Sun HJ, Xiong XQ, et al. Apelin-13 and APJ in paraventricular nucleus contribute to hypertension via sympathetic activation and vasopressin release in spontaneously hypertensive rats. Acta Physiol (Oxf) 2014;212:17-27.

20. Lv X, Li K, Hu Z. Autophagy and Others Respiratory Diseases. Adv Exp Med Biol 2020;1207:585-97.

21. Tian J, Popal MS, Zhao Y, et al. Interplay between 
Exosomes and Autophagy in Cardiovascular Diseases: Novel Promising Target for Diagnostic and Therapeutic Application. Aging Dis 2019;10:1302-10.

22. Du J, Zhang C, Zhao W. Autophagy and Hypertension. Adv Exp Med Biol 2020;1207:213-6.

23. Du J, Li Y, Zhang C. Application of Autophagy in Cardiovascular Diseases. Adv Exp Med Biol 2020;1207:265-70.

24. Gu S, Li X. Regulation of Autophagy in Cardiovascular Diseases by Natural Products. Adv Exp Med Biol 2020;1207:731-6.

25. Hughes WE, Beyer AM, Gutterman DD. Vascular autophagy in health and disease. Basic Res Cardiol 2020;115:41.

26. Zhai C, Shi $W$, Feng $W$, et al. Activation of AMPK prevents monocrotaline-induced pulmonary arterial hypertension by suppression of NF-kB-mediated autophagy activation. Life Sci 2018;208:87-95.

27. Yamanaka R, Hoshino A, Fukai K, et al. TIGAR reduces smooth muscle cell autophagy to prevent pulmonary hypertension. Am J Physiol Heart Circ Physiol 2020;319:H1087-96.

28. Kroemer G, Marino G, Levine B. Autophagy and the integrated stress response. Mol Cell 2010;40:280-93.

29. Liu TY, Xiong XQ, Ren XS, et al. FNDC5 alleviates hepatosteatosis by restoring AMPK/mTOR-mediated autophagy, fatty acid oxidation, and lipogenesis in mice. Diabetes 2016;65:3262-75.

30. Xue N, Lai F, Du T, et al. Chaperone-mediated autophagy degradation of IGF-1Rb induced by NVP-AUY922 in pancreatic cancer. Cell Mol Life Sci 2019;76:3433-47.

31. Kim TW, Cheon C, Ko SG. SH003 activates autophagic cell death by activating ATF4 and inhibiting G9a under hypoxia in gastric cancer cells. Cell Death Dis 2020;11:717.

32. Wang Y, Zhang H, Pang T, et al. Rapamycin improves renal injury induced by Iodixanol in diabetic rats by deactivating the mTOR/p70S6K signaling pathway. Life

Cite this article as: $\mathrm{Xu} \mathrm{T}$, Jia J, Xu N, Ye C, Zheng F, Yuan Y, Zhu GQ, Zhan YY. Apelin receptor upregulation in spontaneously hypertensive rat contributes to the enhanced vascular smooth muscle cell proliferation by activating autophagy. Ann Transl Med 2021;9(8):627. doi: 10.21037/atm-206891
Sci 2020;259:118284.

33. Zhao X, Liu L, Jiang Y, et al. Protective Effect of Metformin against Hydrogen Peroxide-Induced Oxidative Damage in Human Retinal Pigment Epithelial (RPE) Cells by Enhancing Autophagy through Activation of AMPK Pathway. Oxid Med Cell Longev 2020;2020:2524174.

34. Wang D, Uhrin P, Mocan A, et al. Vascular smooth muscle cell proliferation as a therapeutic target. Part 1: molecular targets and pathways. Biotechnol Adv 2018;36:1586-607.

35. Hadrava V, Kruppa U, Russo RC, et al. Vascular smooth muscle cell proliferation and its therapeutic modulation in hypertension. Am Heart J 1991;122:1198-203.

36. Ren XS, Tong Y, Ling L, et al. NLRP3 gene deletion attenuates angiotensin II-induced phenotypic transformation of vascular smooth muscle cells and vascular remodeling. Cell Physiol Biochem 2017;44:2269-80.

37. Mitchell GF. Arterial stiffness and hypertension: chicken or egg? Hypertension 2014;64:210-4.

38. Wang M, Kim SH, Monticone RE, et al. Matrix metalloproteinases promote arterial remodeling in aging, hypertension, and atherosclerosis. Hypertension 2015;65:698-703.

39. Sun HJ, Ren XS, Xiong XQ, et al. NLRP3 inflammasome activation contributes to VSMC phenotypic transformation and proliferation in hypertension. Cell Death Dis 2017;8:e3074.

40. Liu QF, Yu HW, You L, et al. Apelin-13-induced proliferation and migration induced of rat vascular smooth muscle cells is mediated by the upregulation of Egr-1. Biochem Biophys Res Commun 2013;439:235-40.

41. Tai S, Hu XQ, Peng DQ, et al. The roles of autophagy in vascular smooth muscle cells. Int J Cardiol 2016;211:1-6.

42. He L, Zhou Q, Huang Z, et al. PINK1/Parkin-mediated mitophagy promotes apelin-13-induced vascular smooth muscle cell proliferation by AMPKa and exacerbates atherosclerotic lesions. J Cell Physiol 2019;234:8668-82. 
Supplementary

Table S1 Primers for real-time quantitative PCR analysis

\begin{tabular}{lll}
\hline & Primer & Sequences \\
\hline Apelin & Forward & 5'-TCTGGCTCTCCTTGACTG-3' \\
& Reverse & 5'-AGGCATGGGTCCCTTATG-3' \\
APJ & Forward & 5'-GGAAGATGATGGTTACAACTAC-3' \\
& 5'-AGGCTGGCAATGAAGATG-3' \\
GAPDH & Forward & 5'-AGGTCGGTGTGAACGGATTTG-3' \\
& Reverse & 5'-TGTAGACCATGTAGTTGAGGTCA-3' \\
\hline
\end{tabular}

APJ, apelin receptor.

Table S2 Antibodies for Western blot or immunohistochemistry

\begin{tabular}{lll}
\hline & No. & Source \\
\hline Anti-apelin & DF13350 & Affinity Biosciences, Cincinnati, OH, USA \\
Anti-APJ & $20341-1-$ AP & Protein Tech Group Inc, Chicago, IL, USA \\
Anti-PCNA & $10205-2-$ AP & Protein Tech Group Inc, Chicago, IL, USA \\
Anti-GAPDH & $60004-1-$ Ig & Protein Tech Group Inc, Chicago, IL, USA \\
Anti- $\beta$-actin & 4970 & Cell Signaling Technology, Beverly, MA, USA \\
Anti-LC3B & 3868 & Cell Signaling Technology, Beverly, MA, USA \\
Anti- $\alpha$-SMA & 48938 & Cell Signaling Technology, Beverly, MA, USA \\
Anti-ATG5 & 12994 & Cell Signaling Technology, Beverly, MA, USA \\
Anti-p62 & 5114 & Cell Signaling Technology, Beverly, MA, USA \\
\hline
\end{tabular}

APJ, apelin receptor; PCNA, proliferating cell nuclear antigen; LC3B, microtube-associated protein-1 light chain-3B; $\alpha$-SMA, $\alpha$-smooth muscle actin; ATG5, autophagy related protein 5 . 